\title{
Cross-linguistic categorisation of the body: Introduction
}

\author{
N.J. Enfield ${ }^{\mathrm{a}, *}$, Asifa Majid ${ }^{\mathrm{a}}$, Miriam van Staden ${ }^{\mathrm{b}}$ \\ a Max Planck Institute for Psycholinguistics, Postbus 310, 6500 AH Nijmegen, The Netherlands \\ b Amsterdam Center for Language and Communication, University of Amsterdam, General Linguistics, \\ Spuistraat 210, 1012 VT Amsterdam, The Netherlands
}

\begin{abstract}
The domain of the human body is an ideal focus for semantic typology, since the body is a physical universal and all languages have terms referring to its parts. Previous research on body part terms has depended on secondary sources (e.g. dictionaries), and has lacked sufficient detail or clarity for a thorough understanding of these terms' semantics. The present special issue is the outcome of a collaborative project aimed at improving approaches to investigating the semantics of body part terms, by developing materials to elicit information that provides for cross-linguistic comparison. The articles in this volume are original fieldwork-based descriptions of terminology for parts of the body in ten languages. Also included are an elicitation guide and experimental protocol used in gathering data. The contributions provide inventories of body part terms in each language, with analysis of both intensional and extensional aspects of meaning, differences in morphological complexity, semantic relations among terms, and discussion of partonomic structure within the domain. (C) 2005 Elsevier Ltd. All rights reserved.
\end{abstract}

Keywords: Body part terms; Partonomy; Meronymy; Semantic fields; Elicitation technique; Semantic typology; Intensional meaning; Extensional meaning

\footnotetext{
* Corresponding author.

E-mail addresses: nick.enfield@mpi.nl (N.J. Enfield), Asifa.Majid@mpi.nl (A. Majid), m.vanstaden@uva.nl (M. van Staden).
} 


\section{Introduction}

The body is a unique object in human experience, posing a special problem in perception and cognition. It is on the one hand part of our selves, and on the other hand one of the things in the world we encounter. In contrast to ordinary objects, the body affords dual access. It can be seen and touched like any other object, and it can be felt through proprioception and somesthetic inputs. Our bodies are central to daily existence. We are all the time utilising and accommodating to our physical selves, and we regularly encounter conventional linguistic references to the body and its parts. As an environmental universal, the human body is a rich resource for exploring fundamental issues about the nature of categorisation. How similar, or different, are cognitive categories? How do people of radically different cultures categorise and conceptualise the very same world? What role does language play?

Our more specific questions concerning categorisation and linguistic/conceptual segmentation of the body include the following: How do languages conventionally segment the body into parts? Does the set of body part terms constitute a structured system in all languages? Is there a universal, cross-linguistically consistent way of categorising the body? The answers are of some consequence. It has been claimed, for example, that there are universal tendencies for specific body part concepts to serve as source domains for conceptualisation and expression of other aspects of the world, for example spatial location and topological relationships (Svorou, 1993; Heine, 1997). It is widely known that terms for body parts like 'head', 'belly', and 'back' are used in conventional spatial descriptions in many languages. But in order to assess the claim of true universality in such uses, we need to know if the putatively basic source meanings of such terms really are the same across languages. Studies presented in this special issue suggest that there are significant differences.

\section{Semantic typology}

Much work in linguistic typology presupposes the availability and comparability of descriptive materials in a broad range of areally, genealogically and typologically distinct languages. This is valid for certain topics of research, for example constituent order patterns, since these are likely to be adequately covered in any standard grammatical description. However, due to intrinsic limitations of traditional linguistic description, there is little if any detailed or comprehensive information, even from the best grammars and dictionaries, on many aspects of linguistic organisation. The problem is most acute in the domain of semantics. Comparative work in semantics requires explicit language-specific argumentation, of greater detail than is provided in general linguistic descriptions. An example of a critical analytic distinction which requires case-by-case investigation is the status of a linguistic expression as either ambiguous (having numerous distinct meanings) or semantically general (vague). (See below for further discussion of this point.)

Rather than doing comparative semantics by first collecting pre-existing data sources (e.g. dictionaries) and then posing comparative research questions to which these sources should provide answers, it is desirable if not necessary to begin by formulating the research questions and preparing appropriate methods for investigating those questions before undertaking primary data collection in the field. We stress that we are talking about methods in the plural. Each contributor to this special issue conducted first-hand field 
investigation into the body part domain using the same array of multiple techniques. In addition to their own knowledge of the languages, and their own previously collected data and otherwise available sources, contributors developed and implemented two methods of data collection specific to this project. One source of information was an 'Elicitation Guide on Parts of the Body', which first appeared in the 2001 Field Research Manual of the Max Planck Institute for Psycholinguistics Language \& Cognition Group (MPI, L \& C Group). A revised version appears as Enfield (this volume). This provided a common set of points for each researcher, a common focus for elicitation of vocabulary items and certain aspects of their meaning. A second source of information was a 'Body colouring task', developed after preliminary data had been collected from the elicitation guide. This was published in the 2003 Field Research Manual of the MPI, L \& C Group. A revised version appears as van Staden and Majid (this volume). This task provided a common denotational frame, allowing field researchers to directly compare speaker judgements of the extensions of language-specific terms. The two types of task provide different kinds of information about the meanings of body part terms. Combined, they provide a more thorough understanding of the semantics of body part terms than could be gleaned from consulting a traditional grammar or dictionary.

The value of having a group of investigators collectively develop and implement these complementary approaches is twofold. First, each investigator acquires relevant information both from within the language (in elicitation) and without the language (using a nonlinguistic stimulus). The combination of intensional and extensional information enables more thorough understanding of the results. Second, that all the investigators use the same protocol maximises comparability of results. This process of collaborative development and implementation of a research investigation in a specific semantic domain enables a new standard of comparative work toward a discipline of semantic typology.

Since investigations of appropriate depth presuppose expert knowledge of a given language, the only practical way to work (given each individual's limited time and resources) is in a team of scholars, each of whom have language expertise and ongoing access to field research sites. Listed below are the names of the researchers who produced articles for this special issue. Each of them participated in the process of formulating the research questions on linguistic categorisation of the body and its parts, designing multiple methods for their investigation in field work, and travelling to far flung field sites to carry out these investigations first-hand ${ }^{1}$ :

Niclas Burenhult

N.J. Enfield

Alice R. Gaby

Stephen C. Levinson

Asifa Majid

Sergio Meira

Jennie E. Pyers

Angela Terrill

Miriam van Staden

Claudia Wegener

\author{
Jahai (Malaysia) \\ Lao (Laos) \\ Kuuk Thaayorre (Australia) \\ Yélî Dnye (Papua New Guinea) \\ Punjabi (Pakistan/India) \\ Tiriyó (Brazil/Surinam) \\ American Sign Language (USA) \\ Lavukaleve (Solomon Islands) \\ Tidore (Indonesia) \\ Savosavo (Solomon Islands)
}

\footnotetext{
${ }^{1}$ Each of these studies received partial or total support from the Max Planck Society, which we hereby acknowledge with gratitude.
} 


\section{Analytical distinctions}

Articles in this special issue document conventional means for referring to parts of the body in a set of geographically, genetically, and typologically distinct languages. Little or no attention is paid to literally descriptive expressions in which the meaning of the form is a direct combination of the meaning of its parts. Left arm and area between the nose and lip are literally descriptive. Underarm (i.e. armpit), on the other hand, is not literally descriptive because its meaning is not solely a function of the combination of the meanings 'under' and 'arm'. The word has a conventional meaning which must be listed in the lexicon of English. For any such conventional expression, if it refers to a body part, we assume it designates a body part CATEGORY (Brown, 1958). By comparing languages, we can see how similar, or different, body part categories are cross-linguistically.

Previous work on terms for parts of the body has been concerned with distinguishing between degrees of formal complexity of terms. For example, Brown (1976) and Andersen (1978) both make reference to such distinctions in proposed universal patterns in body part terminology. Brown (1976) states that if there is a label for 'hand' in a language this label will always be an 'unanalysable primary lexeme' (p. 405) while the same does not apply for 'fingernail' (p. 409). The assumption here is that 'basic' cognitive categories are labelled by formally less complex terms. Similar reasoning is suggested by earlier work in other domains of cognitive anthropology and psychology such as Berlin and Kay (1969) on colour terminology, and Rosch et al. (1976) on taxonomy. In order to foresee the formulation and testing of hypotheses as to universality in body part terminology, contributions to this special issue make a formal distinction between SIMPLEX and COMPLEX terms for parts of the body. Some contributors stipulate additional criteria, for instance, that simplex terms should be non-technical terms in every day use, or non-loans.

\section{Meaning of body part terms}

The basic challenge of cross-linguistic comparison in the domain of body part semantics is summed up by Brown (1976, p. 421): 'In order to say that a certain labelling procedure universally pertains to body partonomy, one must first establish just what is labelled.' The problem is to determine both the extension (referential range) of a body part term and the intension (the semantic specifications) that account for that range.

Perceptual experience, especially vision, has been regarded as theoretically primary in body part nomenclature. For Brown, establishing what is labelled by a body part term 'necessarily means discovery of those areas of the perceptual grid... of universal recognition' Brown (1976, p. 421). Similarly, Andersen (1978, p. 338) states that principles of categorisation and nomenclature in the body part domain 'derive from the facility of the human perceptual apparatus to deal with particular attributes (e.g. shape, size, spatial orientation)'. Similar views can be found in the Cognitive Linguistics literature (e.g. Johnson, 1987; Lakoff, 1987). The implicit assumption is that body part categories will be the same in every culture because everyone's perceptual apparatus is the same and everyone's bodies are (more or less) the same. That body part categories are derived from perception is a reasonable hypothesis, but in order to test how substantial a role perception plays in our categories it is still necessary to operationalise which aspects of perception may be important for partitioning the body. One candidate is shape. Visual discontinuities in objects, including the human body, have been claimed to determine segmentation into 
parts (e.g. Marr, 1982; Biederman, 1987). Thus, the leg will be a distinct body part because there is a sharp discontinuity in the image where the leg joins the body.

There are terms in all languages described in this special issue for which perceptual discontinuity is relevant. For example, most report segmentation of the limbs with reference to the discontinuity of joints. However, there is no necessary relationship between a perceptual discontinuity and the naming of a part. Savosavo has a category for leg beginning at the hip joint and encompassing the foot. Punjabi and American Sign Language (ASL) distinguish leg from foot, explicitly recognising the discontinuities of both the hip joint and the ankle. Yélî Dyne also has two terms but unlike Punjabi and ASL it distinguishes upper leg from lower leg but ignores the ankle discontinuity by having a single category for lower leg plus foot. Jahai recognises all three parts, upper leg, lower leg, and foot. An exception to the tendency for terms to encode segmentation of limbs at joints is Tidore yohu, which begins at the foot and ends three-quarters of the way up the thigh where there is no visual discontinuity.

As a further demonstration that visually discernable discontinuities do not necessitate a distinction for linguistic categories is the fact that many languages appear not to have distinct labels for 'hand' and 'arm', having just one term for the whole upper limb (Witkowski and Brown, 1985). But due to a strong conviction of universality in body part naming, some researchers have analysed this single term as polysemous, with two distinct meanings, 'hand' and 'arm', instead of having a general meaning 'whole upper limb from shoulder to finger tip'. By this logic, one might conclude that English leg is polysemous, with two meanings 'upper leg' and 'lower leg' (a conceivable situation). But leg refers to the whole of the upper and lower leg together. By the principle of parsimony, polysemy analyses should be avoided. The burden of claiming polysemy is to explicitly establish it using linguistic tests. By using such tests, Terrill (this issue) establishes that Lavukaleve tau 'limb' is general in meaning and not polysemous (with the meanings 'arm' and 'leg'). It is unknown how many languages have genuine polysemy of a single term that here means 'arm' and there means 'hand'. Standard sources, such as dictionaries, do not provide the information required for distinguishing between a term's status as general or ambiguous.

While perceptual discontinuity may play a role in segmenting body parts such as the limbs, there must be other bases for segmentation. Perceptual discontinuity cannot explain the segmentation encoded by terms for some parts of the face, the belly, chest, back, and internal organs (at least where visual access to internal organs is limited). Previous researchers of body part terms mention perception in general but privilege vision and neglect other sensory modalities through which we obtain information about bodies. Other perceptual cues could be operative in distinguishing body part categories. For example, while the fingers are visually much smaller than the arms, they are tactilely 'bigger'. They take up a greater area of the primary somatosensory cortex than arms do (Penfield and Rasmussen, 1950), and could therefore be said to be more salient than arms. Recent research suggests that joint information represented by the motor cortex may also provide a basis for body part segmentation (de Vignemont et al., 2005). Unlike in a visual account, this approach emphasises the primacy of action and the use of joint information in coordinating action.

Apart from properties derived from perception, whether from vision, proprioception, or action, parts are also characterised by functional properties (McClure, 1975, p. 84; Wierzbicka, 1980, 1996; Wilkins, 1996, pp. 277-278; Tversky and Hemenway, 1984; Tversky et al., 2002). A leg is long, vertically oriented and attached to the trunk, but at 
the same time it is used for support and for walking. The nose is for smelling and breathing, eyes are for seeing, ears are for hearing. While the faculty of hearing is not a visually perceivable property of the ear, the concept of hearing is arguably indispensable for the concept 'ear' (Wierzbicka, 1996, 219). Knowing what an 'ear' is means more than simply associating the signifier ear with a perceptual image of an ear. Just as important as the shape, location, and visually perceivable boundaries of an ear is what it does, what it is for (Morrison and Tversky, 1997).

Appearance and function are typically presumed to be related. It is also typically assumed that perception is primary, and that function is therefore inferred from appearance (Tversky et al., 2002). But is this the case for all parts of the body? For many people, human internal organs are never visually perceived. In addition, non-visual modes of perception of many internal organs do not provide a sufficient degree of precision in determining how many or what organs there are. Most people only see illustrations of human internal organs or see their analogues among the organs of butchered animals. Many English speakers may have no visual image of the spleen or appendix, despite these being real parts of the body to which linguistic reference is made. Semantically encoded information concerning relations, functions, and other propositional import is part of a more general component of semantics that may be called the internal logic (Wierzbicka, 1985) or cognitive content (Prinz, 2002) of a linguistic item's meaning. Any given body part term may be linked to perceptual imagery but must denote conceptual or descriptive content, whether the body part it denotes is perceptually well-bounded (such as a fingernail), less well-bounded (such as the cheek), out of sight (such as the spleen) or not perceptually accessible in any way (such as Punjabi $k o D D i$ 'organ in chest cavity deemed to be responsible for sickness'; Majid, this volume).

Many languages in this volume feature terms derived from actions performed with a particular part of the body. The Lao term khaa3 phapl 'back of knee' literally means 'fold leg'. Some Tidore terms are nominalisations of action verbs, e.g. lao ma-jobi 'eyelid' from cobi 'to wink'; $d u b u$ 'fist' from $t u b u$ 'to fight, to pound'. Also, several languages report single terms denoting parts of the body and corresponding bodily processes or products related to a part or process. For example, in Jahai a single term refers to 'belly' (external), 'bowels' (internal), 'shit' and 'to shit'. Kuuk Thaayorre similarly has a single term for 'bum' and 'shit' (cf. Wilkins, 1996).

A final, key issue for research on linguistic categories is how to reconcile the apparent population-level convergence of linguistic meanings with the existence of concepts in the heads of individuals. According to neo-empiricists like Barsalou (1999) and Prinz (2002), concepts are ultimately derived from experience (i.e. perception). Thus, as discussed above, individuals can use structure in the environment to segment and categorise the body. But how are these categories made public such that there is convergence in word meaning? It may be that structural regularities in the environment allow individuals to converge in their segmentation and categorisation of that structure. Accordingly, simulations with individual artificial agents who receive perceptual input of a scene show a degree of coordination of internal representations between those individuals. But the convergence of internal representations is greatly increased when the artificial agents are able to 'talk' to one another, i.e. to use public signs or 'words' to refer to their perceptual experience (Steels, 2003). There are parallels here with categorisation of the body. Visual discontinuities and other perceptual cues can help in segmentation of the body, but an individual has to learn the linguistic conventions of their community in order to be able to ascertain just 
which discontinuities are meaningful (i.e. are picked out by linguistic terms). We hope that data of the kind presented in this special issue will contribute to our understanding of this process.

\section{Anatomical nomenclature as a system}

Does the inventory of body parts and related terms in a language constitute a structured system? Upon what principles could such a system be organised? It is well established that there are sets of words for which the conceptual relation KIND is a basis for systematic and hierarchically nested relations (Lyons, 1977; Wierzbicka, 1985; Cruse, 1986, inter alia). Words in such systems stand in relationships of relative semantic generality, giving rise to transitivity, such that if an $\mathrm{X}$ is a (kind of) $\mathrm{Y}$ and a $\mathrm{Y}$ is a (kind of) $\mathrm{Z}$, then an $\mathrm{X}$ is a (kind of) Z. Thus, a Siamese is a cat, a cat is an animal, therefore a Siamese is an animal. Work in the domain of taxonomic relations has had considerable success, but as data presented in contributions to this special issue suggest, and despite the hopes of some (e.g. Brown, 1976; Andersen, 1978), the specifics of the structural analysis of taxonomic semantics do not transfer straightforwardly to the domain of partonomic relations. As McClure (1975) notes, transitivity does not reliably apply in body partonomy (cf. Cruse, 1986, 2004). She writes, 'teeth are parts of mouths, mouths are parts of faces, but teeth are not parts of faces' (McClure, 1975, p. 84), attributing this failure of transitivity to polysemy of the relation 'part of'. (See Wierzbicka, 1980, pp. 82-83, for arguments that this might be due instead to polysemy of mouth.) It is not clear what relations pertain among sets of terms whose common feature is that they are all part of the same larger whole entity (Palmer and Nicodemus, 1985, p. 345).

There are two relational levels of interest in this domain. First is the relation between the topmost node of the partonomy (the body) and anything that is considered a part of it (by reference to a frame along the lines of English ' $\mathrm{X}$ is a part of $\mathrm{Y}$ '). In this special issue, the domain of the body includes all those things which can be said, by speakers of the language concerned, to be part of the body (or other equivalent locution). A distinction is made between body parts proper and other terms which may be related to the body, but are not part of the body, such as bodily products (snot, piss, shit, etc.) and things on bodies (moles, scars, wrinkles, etc.). Whether something is conceptualised as part of the body (as opposed to merely related to the body) is language-specific. Nevertheless, most contributors have listed and discussed terms in the latter categories.

Many have claimed that all languages have a term meaning 'body' (Brown, 1976; Andersen, 1978; Wierzbicka, 1996; Goddard, 2001), but this is not uncontroversial. Evans and Wilkins (2001) and Wilkins (1996), for example, state that terms denoting the body are diachronically unstable, and that these terms are often polysemous, being used to refer to skin, trunk, and person, as well as to body. Wilkins (1996) argues that a term for person or human being is a better superordinate term or unique beginner, as it does appear to be universally named and is less likely to be polysemous. Contributors to this special issue have established the appropriate unique beginner for their languages based on internal linguistic evidence. So, while Lao, Punjabi and Yélî Dyne have 'body' as a unique beginner, Kuuk Thaayorre, Lavukaleve, Tidore and Savosavo have 'person'.

On the second relational level of interest are relations AMONG elements of the set of parts of the body. Both Brown (1976) and Andersen (1978) want to relate body parts to one another through a hierarchical partonomy. But the discussion of how to construct such 
a hierarchy is unsatisfactory. For example, Brown defines a parton as something which is 'part of an entity and is described as "possessed by" that entity' (Brown, 1976, p. 401), shifting between 'part of' and 'possessed by' as if the two were equivalent (cf. Palmer and Nicodemus, 1985, p. 344, for the same problem). As Swanson and Witkowski (1977, p. 324) point out, 'some writers in the literature have been too eager in placing the anatomical domain into neat, cut and dried, structures with the appropriate (and perhaps culturally and universally obvious) branchings and nicely labelled levels'. While Brown states that the relationship $\mathrm{X}$ possesses $\mathrm{Y}$ is 'an apparent logical inverse of "part of" " (Brown, 1976, p. 422), it is not the case that all relationships of X possesses Y are also relationships of $\mathrm{Y}$ is a part of $\mathrm{X}$. For example, my sports car and my older brother are mine (i.e. possessed by me), but neither is part of me.

While Swanson and Witkowski (1977) argue for possession (as opposed to Brown's partonomy) as the relevant relation in anatomical nomenclature, Palmer and Nicodemus (1985) view the notion of possession as 'the ethnographer's, and not one emerging from elicitation or lexical structure' $(1985$, p. 353$){ }^{2}$ They strongly advocate a view of body parts as standing in 'contiguous-locative' relations to each other, 'defined spatially'. Swanson and Witkowski's claim is that 'the inalienable possession of anatomical terms is the principle or basic relation of this domain and the part-whole relation is secondary at most' (Swanson and Witkowski, 1977, p. 325). Expressions like 'my leg' or 'his head' are said to be 'much more appropriate and elicitable expressions in Hopi (or any language) than the reverse relation of "the leg is part of my body" or "the head is part of his body", (Swanson and Witkowski, 1977, p. 325). However, attempts to pin down the concept of possession (as it applies to relations between parts of a physical whole) in a cross-linguistically comparable way prove equally problematic. The grammarian's use of a label 'possessive construction' for a given structure in a description of a given language does not guarantee that the construction encodes (only) possessive semantics. This is a point made in contributions to this special issue (e.g. Burenhult's paper on Jahai). It may be that while the construction is good for describing situations of possession, its semantics are more general than this, also covering other kinds of situation. In addition, the construction may have multiple meanings, encoding possession in some contexts, and some other relation(s) in other contexts.

Related to this, but yet again distinct, is the relation of CONNECTION. Connection is suggested by Brown to be another possible manifestation of the 'part-whole' relationship (e.g. Brown, 1976, p. 406, 407, 420). But again these relations are not semantically identical. If $\mathrm{X}$ is connected to $\mathrm{Y}$, it is not necessarily part of $\mathrm{Y}$. The television can be connected to the wall but not be part of the wall. Moreover, as Pyers (this issue) points out in relation to ASL, the relationship of connection may be reciprocal so that if $\mathrm{X}$ is connected to $\mathrm{Y}$ then $\mathrm{Y}$ is also connected to $\mathrm{X}$ (in conflict with the notion of a hierarchy for the relevant type of relation).

Contributors to this special issue report difficulties in conclusively establishing comprehensive multi-level partonomies for the body part lexicon. None of the languages appear to encode exhaustive partonomic relations within the full system of body part terms.

\footnotetext{
${ }^{2}$ Their comments are aimed at Brown (1976) in a critique based on data from the Salish language Coeur d'Alene, in which location is argued to be the operative semantic relation among body part terms. The case of Hopi-which motivated Swanson and Witkowski's (1977) support of possession as the operative principleappears to be different again.
} 
Nonetheless, Lao, Punjabi, Tiriyó, Tidore and Yélî Dnye show a partonomic hierarchy between a subset of terms. The articles on ASL, Jahai, Lavukaleve, Savosavo and Kuuk Thaayorre suggest that there is no linguistic evidence for a multi-level or nested partonomic conceptualisation between body part terms. Overall, the data in this special issue do not yield broad-ranging systematic levels of embedding of partonomic relations among body part terms of the kind proposed as universal by Brown (1976) and Andersen (1978).

\section{Conclusion}

Contributions to this special issue suggest - not unexpectedly - that linguistic categorisation of the body is subject to both universal and language-specific principles. Many distinctions are made across languages with reference to the same perceptible discontinuities (e.g. joints such as the shoulder or knee). At the same time, there are terms whose semantics and referential range differ across languages.

Among proposed universals in this domain are that there will be distinct terms for 'body', 'head', 'arm', 'eyes', 'nose', and 'mouth'; the terms for 'head', 'trunk', 'arm', and 'leg' will always be "possessed by" 'body'; all languages will have labels for 'finger', 'toe', and 'nail'; and if a language has a distinct term for 'foot', it will have a distinct term for 'hand' (Andersen, 1978; cf. Brown, 1976). We find even in this small sample a number of counterexamples.

Several languages do not have a general term meaning 'body'. In Tidore and Kuuk Thaayorre the highest level in the partonomy would be a word for 'person'. There is no term meaning 'arm' in Lavukaleve, and no term meaning 'mouth' in Jahai. There are languages with more distinctions on lower limbs than on upper limbs. For example, Lavukaleve does not have a simplex term for 'hand' but does for 'foot'. Not all languages appear to favour conventional lexicalisation of parts at the level of the entire limb. Jahai systematically favours a finer level of segmentation. It has terms for 'upper leg', 'lower leg', and 'foot', but no term for 'leg'. Jahai also does not have a word for 'face' or 'mouth', but has more than fifteen simplex terms to label smaller parts, such as the eyes, upper lip, lower lip, teeth, 'prominent ridges on either side of the forehead', and 'wrinkles between the eyebrows'. Some terms have meanings with little or no reference to perceptible phenomena (e.g. Punjabi $k \supset D D i$ 'organ in chest cavity deemed to be responsible for sickness'). In some cases languages differ in whether a particular entity is considered part of the body at all. For instance the 'soul' or 'life force' is considered a body part in Yélî Dnye and Jahai, but not in Punjabi. Further, beyond the extensional range of terms, there are dimensions of meaning with ethnographic importance (such as Lao speakers' beliefs and practices concerning the feet and the head, and the ways in which this affects usage and interpretation of the Lao word tiin3 'foot').

As a final point, we raise the issue of individual differences. Pyers reports inconsistent judgements in ASL regarding terms for major parts of the body such as 'arm'. Burenhult (on Jahai) and Meira (on Tiriyó) report differences between individual speakers relating to extensional meanings of terms for parts of the back. Terrill describes variation in extensional meanings of Lavukaleve 'belly', 'chest', and 'side', and in whether the term for 'head' is said to include 'face'. Wegener provides illustrations from the colouring in task for Savosavo revealing variation in extension of a number of terms, including those for 'face', 'waist', and 'lower thigh'. Majid (on Punjabi) finds another type of individual variation, where generational differences indicate a possible language shift from Punjabi to 
Urdu. Individual variation in linguistic categorisation remains an intriguing and important area for investigation.

The aim of this special issue is to offer primary data on linguistic conventions for categorisation of the human body and its parts in a diverse group of languages, with relevance to a number of theoretical and analytical issues concerning categorisation and linguistic semantics. We offer this collection as a step in reviving interest in the empirical study of the way in which human beings conceptualise and categorise their bodies as physical entities with parts. While much scholarly interest in the study of meaning has presupposed that the human body is a basic pre-linguistic source for conceptual structure (feeding into embodiment, metaphor, semantic extension, etc.), it may be that there are fewer points of convergence across language communities in the concrete vocabulary of the body than previously imagined.

\section{Acknowledgements}

We thank Niclas Burenhult, Jennie Pyers, and the other contributors to this volume for their input to this introduction and to the formulation of the project reported on in this special issue. We are also very grateful to Marloes Verhoeven for her expert and patient assistance in preparing the volume, and Alex Dukers for his work in preparing many of the illustrations.

\section{References}

Andersen, E.S., 1978. Lexical universals of body-part terminology. In: Greenberg, J.H. (Ed.), Universals of Human Language. Stanford University Press, Stanford, pp. 335-368.

Barsalou, L.W., 1999. Perceptual symbol systems. Behavioural and Brain Sciences 22, 577-609.

Berlin, B., Kay, P., 1969. Basic Color Terms. Their Universality and Evolution. University of California Press, Berkeley and Los Angeles.

Biederman, I., 1987. Recognition-by-components: A theory of human image understanding. Psychological Review 94, 115-147.

Brown, R., 1958. How shall a thing be called? Psychological Review 65, 14-21.

Brown, C.H., 1976. General principles of human anatomical partonomy and speculations on the growth of partonomic nomenclature. American Ethnologist 3, 400-424.

Cruse, D.A., 1986. Lexical Semantics. Cambridge University Press, Cambridge.

Cruse, A., 2004. Meaning in Language: An Introduction to Semantics and Pragmatics. Oxford University Press, Oxford.

de Vignemont, F., Tsakiris, M., Haggard, P., 2005. Body mereology. In: Knoblich, G., Thornton, I.M., Grosjean, M., Shiffrar, M. (Eds.), Perception of the Human Body from the Inside Out. Oxford University Press, Oxford.

Enfield, N.J., this volume. Elicitation guide on parts of the body. In: Majid, A., Enfield, N.J., van Staden, M. (Eds.), Parts of the Body: Cross-Linguistic Categorisation (Special Issue). Language Sciences, doi:10.1016/ j.langsci.2005.11.003.

Evans, N., Wilkins, D.P., 2001. The complete person: networking the physical and the social. In: Simpson, J., Nash, D., Laughren, M., Austin, P., Alpher, B. (Eds.), Forty Years on: Ken Hale and Australian Languages. Pacific Linguistics 512, Canberra, pp. 493-521.

Goddard, C., 2001. Lexico-semantic universals: a critical overview. Linguistic Typology 5, 1-65.

Heine, B., 1997. Cognitive Foundations of Grammar. Oxford University Press, Oxford.

Johnson, M., 1987. The Body in the Mind. The Bodily Basis of Meaning, Imagination, and Reason. The University of Chicago Press, Chicago.

Lakoff, G., 1987. Women, Fire and Dangerous Things: What Categories Reveal about the Mind. Chicago University Press, Chicago.

Lyons, J., 1977. Semantics. Cambridge University Press, Cambridge. 
Majid, A., this volume, Body part categorisation in punjabi. In: Majid, A., Enfield, N.J., van Staden, M. (Eds.), Parts of the Body: Cross-Linguistic Categorisation (Special Issue). Language Sciences, doi:10.1016/ j.langsci.2005.11.012.

Marr, D., 1982. Vision. W.H. Freeman and Company, New York.

McClure, E.F., 1975. Ethno-anatomy: the structure of the domain. Anthropological Linguistics 17, 78-88.

Morrison, J.B., Tversky, B., 1997. Body schemas. In: Proceedings of the Nineteenth Annual Conference of the Cognitive Science Society. Lawrence Erlbaum Associates, Stanford University, Stanford.

Palmer, G.B., Nicodemus, L., 1985. Coeur d'Alene exceptions to proposed universals of anatomical nomenclature. American Ethnologist 12, 341-359.

Penfield, W., Rasmussen, T., 1950. The Cerebral Cortex of Man. Maxmillan, New York.

Prinz, J., 2002. Furnishing the Mind: Concepts and their Perceptual Basis. MIT Press, Cambridge, MA.

Rosch, E., Mervis, C.B., Gray, W.D., Johnson, D.M., Boyes-Braem, P., 1976. Basic objects in natural categories. Cognitive Psychology 8 (3), 382-439.

Steels, L., 2003. Evolving grounded communication for Robots. Trends in Cognitive Sciences 7, 308-312.

Svorou, S., 1993. The Grammar of Space. John Benjamins Publishing Company, Amsterdam.

Swanson, R.A., Witkowski, S., 1977. Hopi ethnoanatomy: a comparative treatment. Proceedings of the American Philosophical Society 121, 320-337.

Tversky, N., Hemenway, K., 1984. Objects, parts and categories. Journal of Experimental Psychology: General $113,169-193$.

Tversky, B., Morrison, J.B., Zacks, J.M., 2002. On bodies and events. In: Prinz, M.A. (Ed.), The Imitative Mind. Cambridge University Press, Cambridge, pp. 135-152.

van Staden, M., Majid, A., this volume. Body colouring task. In: Majid, A., Enfield, N.J., van Staden, M. (Eds.), Parts of the Body: Cross-Linguistic Categorisation (Special Issue). Language Sciences, doi:10.1016/ j.langsci.2005.11.004.

Wierzbicka, A., 1980. Lingua Mentalis. Academic Press, Sydney.

Wierzbicka, A., 1985. Lexicography and Conceptual Analysis. Karoma, Ann Arbor.

Wierzbicka, A., 1996. Semantics: Primes and Universals. Oxford University Press, Oxford.

Wilkins, D.P., 1996. Natural tendencies of semantic change and the search for cognates. In: Durie, M., Ross, M. (Eds.), The Comparative Method Reviewed: Regularity and Irregularity in Language Change. Oxford University Press, Oxford, pp. 264-304.

Witkowski, S.R., Brown, C.H., 1985. Climate, clothing, and body-part nomenclature. Ethnology 24, 197-214. 